\title{
Continuum contribution to excitonic four-wave mixing due to interaction-induced nonlinearities: A numerical study
}

Sayed, Karim El; Birkedal, Dan; Vadim, Lyssenko; Hvam, Jørn Märcher

Published in:

Physical Review B

Link to article, DOI:

10.1103/PhysRevB.55.2456

Publication date:

1997

Document Version

Publisher's PDF, also known as Version of record

Link back to DTU Orbit

Citation (APA):

Sayed, K. E., Birkedal, D., Vadim, L., \& Hvam, J. M. (1997). Continuum contribution to excitonic four-wave mixing due to interaction-induced nonlinearities: A numerical study. Physical Review B, 55(4), 2456-2465. https://doi.org/10.1103/PhysRevB.55.2456

\section{General rights}

Copyright and moral rights for the publications made accessible in the public portal are retained by the authors and/or other copyright owners and it is a condition of accessing publications that users recognise and abide by the legal requirements associated with these rights.

- Users may download and print one copy of any publication from the public portal for the purpose of private study or research.

- You may not further distribute the material or use it for any profit-making activity or commercial gain

- You may freely distribute the URL identifying the publication in the public portal 


\title{
Continuum contribution to excitonic four-wave mixing due to interaction-induced nonlinearities: A numerical study
}

\author{
K. El Sayed \\ Department of Physics, University of Florida, Gainesville, Florida 32611 \\ D. Birkedal, V. G. Lyssenko, ${ }^{*}$ and J. M. Hvam \\ Mikroelektronik Centret, The Technical University of Denmark, Building 345 East, DK-2800 Lyngby, Denmark
}

(Received 20 May 1996; revised manuscript received 19 August 1996)

\begin{abstract}
We present a theoretical investigation of ultrafast transient four-wave mixing (FWM) of GaAs quantum wells for coherent excitation of excitons and a large number of continuum states. It is shown that in this case the line shape of the FWM signal is drastically altered due to an interaction-induced coupling of the exciton to all the excited continuum states. The signal is dominantly emitted at the spectral position of the exciton and decays, as a function of delay, on a time scale set by the duration of the laser pulse rather than by the intrinsic dephasing time. Nevertheless, the spectral width of the exciton line in the FWM spectrum and in the decay of the time-resolved FWM signal in real time are governed by the intrinsic excitonic dephasing rate. It is shown that for pulse durations of $\sim 100 \mathrm{fs}$ (for GaAs quantum wells) this behavior can be explained as the influence of the Coulomb exchange interaction, while for even shorter pulses this behavior is dominantly caused by nonlinear polarization decay. [S0163-1829(97)07404-3]
\end{abstract}

\section{INTRODUCTION}

At low excitation densities and low temperatures the optical response near the band gap of a direct gap semiconductor is dominated by the mutual Coulomb interaction of the excited carriers. The Coulomb interaction leads to the formation of excitons, coherent exchange effects, and, further, to incoherent scattering and screening. ${ }^{1}$

Transient four-wave mixing (FWM) experiments have proven to be a valuable tool for the investigation of these many-body effects. In FWM experiments the semiconductor sample is excited by two laser pulses propagating in slightly different directions $\vec{K}_{1}$ and $\vec{K}_{2}$. The second pulse is delayed with respect to the first by a time $\tau_{o}$. The interference pattern of the two beams sets up a transient grating, which, due to nonlinear effects, diffracts other photons from the beams. The FWM signal is recorded in the background-free direction, $2 \vec{K}_{2}-\vec{K}_{1}$.

Simulations of FWM experiments are mostly based on the nonlinearities due to Pauli blocking and coherent Coulomb exchange effects. Due to the large numerical effort involved in these simulations, the effect of scattering, i.e., polarization decay and carrier relaxation, is modeled only on a simple phenomenological level (relaxation time approximations). However, many features of FWM signals can be understood within this framework. ${ }^{2-5}$

One prediction of this approach is that both the spectral width and the decay of the FWM signal in delay time at the excitonic resonance is governed by the intrinsic excitonic dephasing rate. In recent experiments, it has been shown that this is not true if, together with the exciton, a large number of continuum states are coherently excited. ${ }^{6}$ In this case the decay of the excitonic FWM signal, as a function of delay $\tau_{o}$, is significantly faster than the dephasing time of the excitons. ${ }^{7-10}$ However, the width of the spectrally resolved excitonic FWM response and the real time evolution of the FWM signal reflect the intrinsic excitonic dephasing time. Furthermore, virtually no signal can be observed at the spectral position of the resonantly excited continuum states. ${ }^{7,10}$ We show here that the excitonic FWM signal consists of two contributions: (i) an intense spectrally narrow part only present for short time delays and (ii) a weak exponentially decaying part reflecting the intrinsic coherence properties of the exciton states. The prompt intense part of the signal is caused by the nonlinear coupling of the continuum states to the exciton due to the Coulomb interaction. In the following we refer to the prompt intense part as the continuum contribution (CC).

For excitations with laser pulses with $\sim 100$ fs duration in GaAs quantum wells tuned 10-20 meV above the exciton, the bandwidth of the coherently excited continuum states is on the order of the excitonic binding energy. In this case the $\mathrm{CC}$ has been described in terms of a formation of an excitonic wave packet by Feldmann et al. ${ }^{8}$ The authors attributed the decay of the strong initial CC in the FWM signal to a destructive interference between the coherently excited microscopic polarizations. However, we showed experimentally in Ref. 7 that CC effects are absent if the excited excitons and continuum states belong to different quantum wells. Thus, $\mathrm{CC}$ is not caused by a interference of the polarizations from independent transitions. We show here that $\mathrm{CC}$ is an interaction effect, well explained by the influence of the coherent Coulomb exchange interaction. At low densities this interaction changes the optical response of the semiconductor from that of an ensemble of independent two-level atoms to that of an exciton system, i.e., a multilevel atom. Indeed, Feldmann et al. reported that their experimental results can be explained with a multilevel model.

CC can also be observed when the bandwidth of the coherently excited continuum states is much larger than the excitonic binding energy. ${ }^{10} \mathrm{We}$ show that the $\mathrm{CC}$ in this case 
is caused by nonlinear polarization decay: In the low-density regime as well as for ultrashort times the additional dephasing due to carrier-carrier scattering is linear in the induced carrier density. The optical polarization therefore decays faster in an intensity maximum than in an intensity minimum. This causes an additional FWM signal. ${ }^{11,12}$ An exciton, i.e., the polarization, can be scattered off virtually any carrier and therefore, in this nonlinear process, the partial transient gratings associated with each carrier state add up coherently (see Sec. III). It is shown that the partial transient gratings add constructively for zero delay, giving rise to a strong enhancement of the FWM signal. ${ }^{11-13}$ Due to the continuous spectral distribution of the carrier states the sum over the partial transient gratings exhibits an inhomogeneous decay as a function of delay, i.e., the additional signal vanishes rapidly. This particular aspect of Coulomb kinetics can easily be included into FWM simulations by assuming that the phenomenological dephasing rate increases linearly with the total induced carrier density. This concept is known as excitation-induced dephasing (EID). EID, i.e., a phenomenological dephasing rate which is a linear function of the total carrier density $n(t)$, is a well-established concept for lowdensity exciton systems,

$$
\gamma(n(t))=\gamma+\gamma^{\prime} n(t) .
$$

Schultheis et al. ${ }^{14}$ determined the density dependence of the dephasing rate from time integrated FWM signals for bulk GaAs and Honold et al. ${ }^{15}$ for quantum wells, as a function of the background density. In these experiments the authors controlled a background density by applying a prepulse, which pumps the system several picoseconds before the two FWM pulses arrive. They reported a linear relation between the decay rate of the FWM signal in delay time and the background density which corresponds to Eq. (1.1) over a wide density range. Kim et al. ${ }^{11}$ performed similar experiments for GaAs quantum wells, but they did not apply a prepulse. Still they found a linear dependence of the decay rate of the FWM signal as a function of the excitation intensity. ${ }^{16}$

In the simulations of FWM signals EID has been used to model the dependence of the FWM signals on the relative polarization of the two exciting laser pulses. ${ }^{17,18}$ An analytic investigation of the influence of EID on FWM signals is given by Wang et al. ${ }^{12}$ They used a two-level atom model similar to the one considered in Sec. III of this paper for their analysis. They reported that EID gives rise to an additional contribution to the FWM signal and that this contribution is similar to the signal due to the Coulomb exchange interaction. Wang et al. based their two-level atom model on an extension of the nonlinear Schödinger equation, proposed in Ref. 19. In this model only a single transition was considered and therefore one of the main aspects of the $\mathrm{CC}$, the interaction of an isolated state with a continuum of carrier states, cannot be included.

This paper is organized as follows. In Sec. II, numerical solutions of the semiconductor Bloch equations with and without EID are given. In Sec. III, we present a simple twolevel atom model with EID, which can be solved analytically. It is shown that the influence of EID on FWM signals can be understood completely within this simplified model.
Discussions and conclusions are given in Sec. IV. In the Appendix we present a motivation of the EID approach.

\section{SEMICONDUCTOR BLOCH EQUATIONS WITH EXCITATION INDUCED DEPHASING}

The optical response of a semiconductor excited by a laser pulse $E(t)$ can be described by the semiconductor Bloch equations (SBE). ${ }^{20-22}$ They are the equations of motion of the occupation numbers $f_{j \mathbf{k}}=\left\langle a_{j \mathbf{k}}^{\dagger} a_{j \mathbf{k}}\right\rangle$ and the microscopic polarization $P_{\mathbf{k}}=\left\langle a_{h \mathbf{k}}^{\dagger} a_{e \mathbf{k}}\right\rangle$. For a simple two-band model the particle index $j$ is $e$ for the electrons and $h$ for the holes. The SBE read:

$$
\begin{aligned}
& \frac{\partial}{\partial t} P_{k}=\left.\frac{\partial}{\partial t} P_{k}\right|_{\text {coh }}+\left.\frac{\partial}{\partial t} P_{k}\right|_{\text {scatt }}, \\
& \frac{\partial}{\partial t} f_{j k}=\left.\frac{\partial}{\partial t} f_{j k}\right|_{\text {coh }}+\left.\frac{\partial}{\partial t} f_{j k}\right|_{\text {scatt }} .
\end{aligned}
$$

The coherent parts of Eq. (2.1) are given by the equations of motion on the Hartree-Fock level:

$$
\begin{aligned}
\left.\frac{\partial}{\partial t} P_{k}\right|_{\mathrm{coh}}= & -i\left[e_{e k}(t)+e_{h k}(t)\right] P_{k}(t) \\
& +i\left[1-f_{e k}(t)-f_{h k}(t)\right] \Omega_{k}^{R}(t), \\
\left.\frac{\partial}{\partial t} f_{j k}\right|_{\text {coh }} & =-2 \operatorname{Im}\left[\Omega_{k}^{R}(t) P_{k}^{*}(t)\right] .
\end{aligned}
$$

The mean-field, or Hartree-Fock part of the carrier-carrier interaction describes the Coulomb exchange interaction. This coherent interaction leads to an additional driving field, the so-called local field, which acts similarly to the applied laser field $E(t)$. It is the local field which causes the formation of excitons. Furthermore, the exchange interaction shifts the free single-particle energies $\hbar \varepsilon_{j k}$. This effect is known as band gap renormalization.

$$
\begin{gathered}
\hbar \Omega_{k}^{R}(t)=\frac{d_{k} E(t)}{2}+\sum_{\mathbf{q}} V_{q} P_{|\mathbf{k}-\mathbf{q}|}(t), \\
\hbar e_{j k}(t)=\varepsilon_{j k}-\sum_{\mathbf{q}} V_{q} f_{j|\mathbf{k}-\mathbf{q}|}(t) .
\end{gathered}
$$

Within the rotating wave approximation, $E(t)$ denotes the pulse envelope of the optical field. $d_{k}$ is the interband optical matrix element. $V_{q}$ is the bare Coulomb potential given by $V_{q}=2 \pi e^{2} / \epsilon_{o} q$ for an ideal quantum well with the background dielectric constant $\epsilon_{o}$. The free single-particle energies are given by

$$
\begin{gathered}
\varepsilon_{e k}=\frac{(\hbar k)^{2}}{2 m_{e}}-\Delta_{o}, \\
\varepsilon_{h k}=\frac{(\hbar k)^{2}}{2 m_{h}} .
\end{gathered}
$$


Here, $\Delta_{o}=\hbar \omega_{o}-E_{g}$ is the detuning between the central frequency $\omega_{o}$ of the pulse and the unrenormalized band gap $E_{g}$.

The SBE have been solved numerically for various approximations for the scattering integrals. Solutions for quantum kinetic ${ }^{23-25}$ and Boltzmann scattering ${ }^{26,27}$ integrals for excitations with only one pulse have been used to investigate the carrier relaxation kinetics and the decay of the induced polarization. The information obtained in a one-pulse simulation is, unfortunately, not directly related to the measured quantities in FWM. For a detailed comparison it is necessary to include the two pulses exciting the sample at different angles and arriving at different times. ${ }^{2,3,28}$ Furthermore, one has to simulate a long time interval in order to obtain reliable spectral information. This increases the numerical effort by about two orders of magnitude. Consequently, only a few FWM simulations are reported which use Boltzmann-like ${ }^{13}$ or even quantum kinetic ${ }^{28}$ scattering integrals. Usually a simple relaxation time approximation for the dephasing rate $\left(\gamma=1 / T_{2}\right)$ is used to approximate the scattering integrals,

$$
\begin{gathered}
\left.\frac{\partial}{\partial t} P_{k}\right|_{\text {scatt }}=-\gamma P_{k}(t), \\
\left.\frac{\partial}{\partial t} f_{j k}\right|_{\text {scatt }}=0 .
\end{gathered}
$$

The set of Eqs. (2.2) and (2.5) has proven to be sufficient for the understanding of many features in FWM. ${ }^{2-5}$ Nevertheless, it fails to predict the strength and shape of the CC to the excitonic FWM signal if a large number of continuum states are excited together with the exciton. ${ }^{7,10}$ We now show that the experimentally observed features of the $\mathrm{CC}$ can be modeled easily by introducing nonlinear polarization decay in the simulation on the phenomenological level of EID, as

$$
\left.\frac{\partial}{\partial t} P_{k}\right|_{\text {scatt }}=-\left(\gamma+\frac{\gamma^{\prime}}{2} \sum_{j=e, h \mathbf{p}} f_{j p}(t)\right) P_{k}(t)
$$

The set of Eqs. (2.1), with (2.2) and (2.6), contains four distinct nonlinear terms which (separately) cause FWM signals. The phase space filling (PSF) nonlinearity is given by $i\left(1-f_{e k}-f_{h k}\right) d_{k} E / 2$. If a given $k$ state is bleached $\left(f_{e k}+f_{h k}=1\right)$ the laser field cannot induce another transition (Pauli blocking). If the state is inverted $\left(f_{e k}+f_{h k}>1\right)$ stimulated emission occurs instead of absorption. In combination with the Pauli blocking term also the local field (LF) contributes to the nonlinear response $\left[i\left(1-f_{e k}-f_{h k}\right) \Sigma_{\mathbf{q}} V_{q} P_{|\mathbf{k}-\mathbf{q}|}\right]$. Furthermore, the polarization of a given $k$-state is also coupled to the occupation numbers by the band gap renormalization (BGR) $\left[i \Sigma_{\mathbf{q}} V_{q}\left(f_{e|\mathbf{k}-\mathbf{q}|}+f_{h|\mathbf{k}-\mathbf{q}|}\right) P_{k}\right]$. A structurally similar coupling of the polarization to the occupation numbers arises from EID $\left[-\gamma^{\prime} / 2 \Sigma_{\mathbf{p}}\left(f_{e p}+f_{h p}\right) P_{k}\right]$.

\section{A. Narrow band width excitation}

We solve the SBE with a density-dependent dephasing rate for an ideal two-dimensional (2D) quantum well in a four-wave mixing geometry. A numerical scheme proposed in Ref. 28 is used. All runs are scaled with $a_{o}=\hbar^{2} \epsilon_{o} / \mu e^{2}$
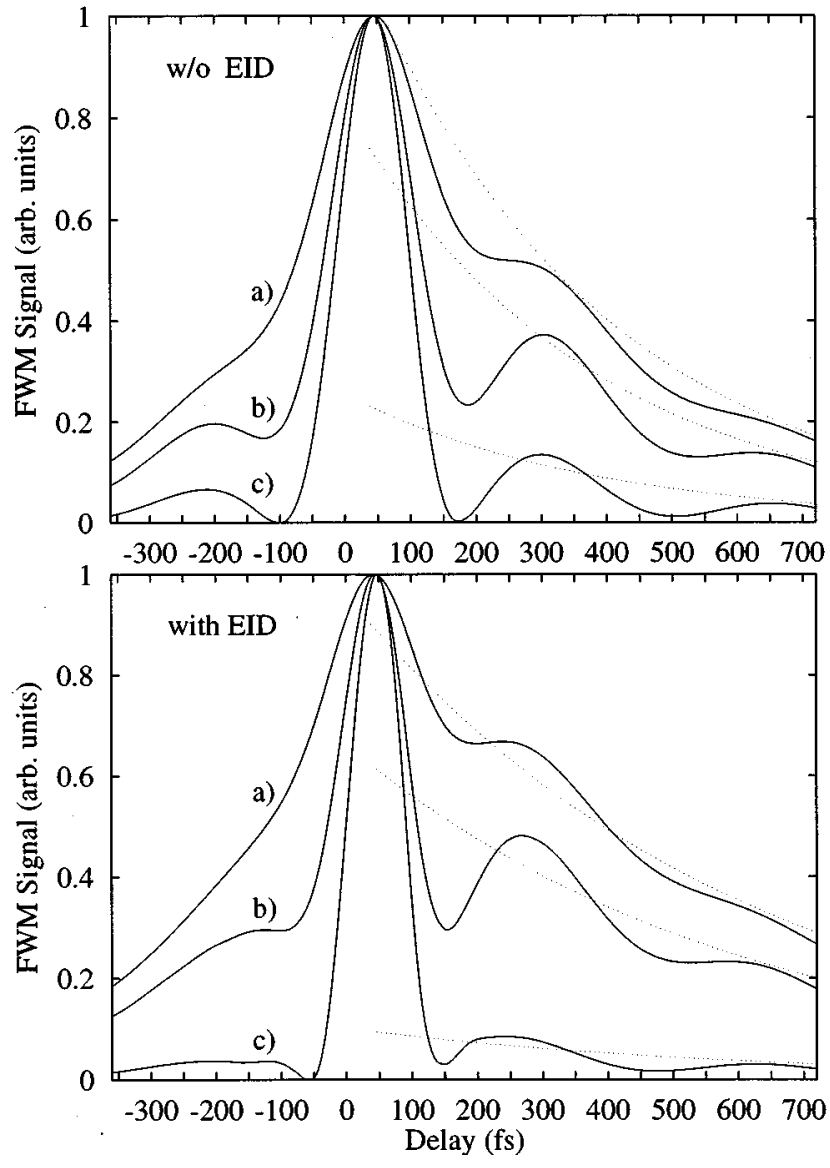

FIG. 1. Four-wave mixing signal at $1 s$ exciton resonance for an excitation with 72 fs pulses vs delay time simulated from the SBE for detunings $\Delta_{o}=\hbar \omega_{o}-E_{g}$ of the central laser frequency from the band edge of of (a) $-E_{o}$, (b) $-0.25 E_{o}$, and (c) $0.5 E_{o}$. Upper: Without EID for $T_{2}=750 \mathrm{fs}$ (solid lines) and exponential decay with $T_{2}=750 \mathrm{fs}$ (dotted lines). Lower: With EID for $T_{2}=1200 \mathrm{fs}$ and $\gamma^{\prime}=3 \times 10^{-13} \mathrm{~cm}^{2} / \mathrm{fs}$ (solid lines) and exponential decay with $T_{2}=1200 \mathrm{fs}$ (dotted lines). The maxima of each curve is normalized to unity for better comparison.

$=14 \mathrm{~nm}$ and $E_{o}=2 \hbar^{2} / \mu a_{o}^{2}=16.8 \mathrm{meV}$, where $\mu=\left(1 / m_{e}\right.$ $\left.+1 / m_{h}\right)^{-1}$. The pulse envelope

$$
E(t)=\mathcal{E}(t) e^{i \vec{K}_{1} \vec{R}}+\mathcal{E}\left(t-\tau_{o}\right) e^{i \vec{K}_{2} \vec{R}}
$$

is the sum of two identical Gaussians $\mathcal{E}(t)$ with an intensity full width half maximum of $72 \mathrm{fs}$. The second pulse is delayed with respect to the first by the time $\tau_{o}$.

Figure 1 shows the resulting excitonic FWM signal for an excitation with a detuning $\Delta_{o}=\hbar \omega_{o}-E_{g}$ of the central laser frequency from the (unrenormalized) band edge of (a) $-E_{o}$, (b) $-0.25 E_{o}$, and (c): $0.5 E_{o}$. The upper part of Fig. 1 shows curves computed without EID ( $\gamma=1 / 750$ fs and $\gamma^{\prime}=0$ ) while we used in the lower part an EID coefficient of $\gamma^{\prime}=3 \times 10^{-13} \mathrm{~cm}^{2} / \mathrm{fs}$. This value corresponds to the experimentally determined value for EID due to nonthermalized carriers, ${ }^{14}$ and to the value reported in Ref. 11. For the calculations with EID we take $\gamma=1 / 1200 \mathrm{fs}$, a value that ensures a similar average dephasing rate in the runs with and without EID. Consequently, we find in the simulations for both dephasing models the same spectral width of the exci- 

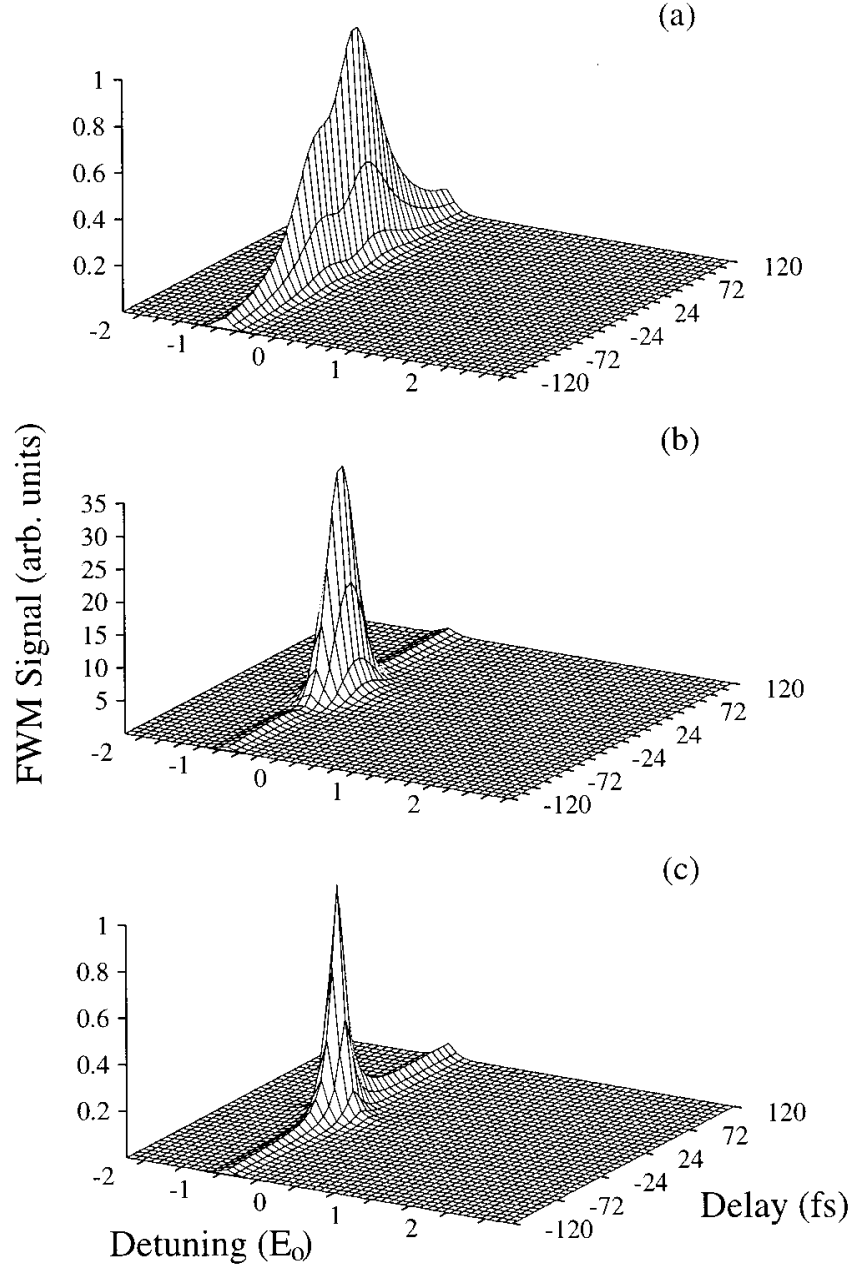

FIG. 2. FWM spectrum for an excitation with $24 \mathrm{fs}$ pulses and a detuning if the central laser frequency from the band edge of $\Delta_{o}=1.5 E_{o}$ vs delay time and detuning $\Delta$ of the photon energy from the band edge computed with the SBE without EID (a) and with EID (b). FWM spectrum on $\chi^{(3)}$ level for an ensemble of two-level atoms with EID (c). (Other parameters are explained in the text.)

tonic FWM signal (not shown). For better comparison the maximum of each curve is normalized to unity. The original maxima are (a) 0.22, (b) 0.1, and (c) 0.003 for the runs without EID and (a) 1.9, (b) 1.1, and (c) 0.12 for the runs with EID. The induced carrier densities in a maximum of the interference pattern of the two beams are (a) $3 \times 10^{9} / \mathrm{cm}^{2}$, (b) $2.6 \times 10^{9} / \mathrm{cm}^{2}$, and (c) $1.4 \times 10^{9} / \mathrm{cm}^{2}$.

For resonant excitation of the continuum [case (c)] a strong initial continuum contribution can be seen, which decays rapidly. After about $150 \mathrm{fs}$ we are left with the intrinsic excitonic signal, which decays $\propto \exp \left(-2 \gamma \tau_{o}\right)$ and exhibits $1 \mathrm{~s}-2 \mathrm{~s}$ beats. (The exponential decay is shown as dotted lines in Fig. 1.) At the same time virtually no signal is emitted at the spectral position of the continuum itself, similar to the data shown in Fig. 2. In case (b) fewer continuum states are excited and we find a smaller enhancement of the FWM signal around zero delay. For resonant excitation of the 1s exciton [case (a)] the signal shows no signs of CC. The inclusion of EID does not change the overall shape of the signal, but increases the signal intensity significantly. The signal computed with EID is 40 times stronger for case (c) than the corresponding one without EID. In case (a) this figure is 8.6 and in case (b) it is 11. One can see also a further temporal narrowing of the prompt part of the signal, due to the broad band nature of the EID induced coupling. The inclusion of EID also increases the ratio of the exponential tail to the initial peak for case (c). Without EID we find a ratio of $1 / 10$, with EID it is $1 / 20$. The PSF nonlinearity contributes to the FWM signal only for positive delay, while interaction induced nonlinearities always contribute. In the later case the main difference is that for positive delay the overall decay constant is $T_{2} / 2$ while for negative delay it is $T_{2}$ /4. [See, e.g., Eq. (3.14) and Refs. 2-4,29.] All FWM signals shown in this paper are of comparable strength for positive and negative delay. This shows that for the cases considered here the signals induced by PSF are of negligible strength compared to the interaction induced ones.

We conclude further that the coherent Coulomb exchange interaction and EID lead to CC in a similar fashion if the bandwidth of the laser excitation is comparable to the exciton binding energy. Feldmann et al. ${ }^{8}$ reported FWM signals from quantum wells computed with the SBE, i.e., including the exchange interaction but without EID. Their results agree completely with the data shown in the upper part of Fig. 1 . They interpreted the fast initial decay of the FWM signal as a destructive interference between the polarizations of the $1 s$ exciton and the excitonic continuum states. However, we would like to point out that CC cannot be explained by an interference of the polarizations from independent transitions as we show in Ref. 7, where FWM signals from a multiquantum-well sample with different well thicknesses $(80 \AA$, $100 \AA, 130 \AA$, and $160 \AA$ ) were investigated. If the laser is tuned to the $1 s$ exciton of the $100 \AA$ A quantum well we did not find $\mathrm{CC}$ at the spectral position of the $100 \AA 1 \mathrm{~s}$ exciton although a large number of continuum states were excited in the neighboring $130 \AA$ quantum well. Nevertheless, at the spectral position of the $130 \AA 1 s$ exciton we saw a pronounced CC. This is an experimental proof that CC is indeed an interaction effect. The coupling induced by the Coulomb exchange interaction is structurally similar to the coupling induced by EID [see Eqs. (2.2), (2.3), and (2.6)] and it leads to $\mathrm{CC}$ is a similar fashion.

\section{B. Wide bandwidth excitation}

In this section we show simulations computed for a laser pulse duration of $24 \mathrm{fs}$ and a detuning of $\Delta_{o}=1.5 E_{o}$. The pulse intensity is chosen such that the maximum induced carrier density at an interference maximum of the two beams is $n=1.4 \times 10^{9} / \mathrm{cm}^{2}$. For the density-independent part of the dephasing rate we now take $\gamma=1 / 250 \mathrm{fs} .{ }^{30}$ In this section figures with the label (a) show simulations without EID, while figures labeled (b) were computed with $\gamma^{\prime}$ $=3 \times 10^{-13} \mathrm{~cm}^{2} / \mathrm{fs}$.

Figures 2(a) and 2(b) show the FWM spectra for the parameter set (a) and (b), respectively. The spectra are scaled such that the maximum of the signal for set (a) is unity. It can be seen that the EID induced signal increases the excitonic FWM signal at zero delay by a factor of $\approx 35$. For nonzero delay the signal decays rapidly. Although the central frequency of the laser is deep in the continuum the FWM 


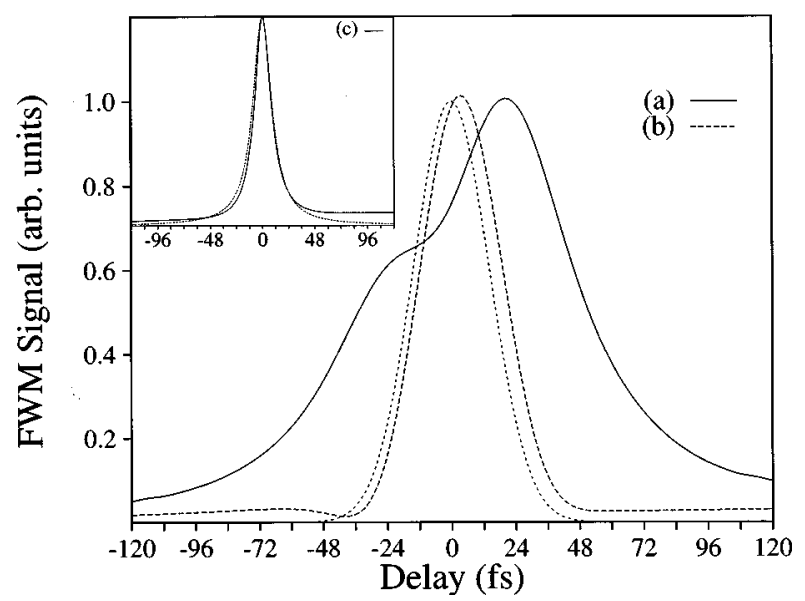

FIG. 3. Cuts from Fig. 2. Excitonic FWM signal vs delay time. For better comparison the maximum of each curve is normalized to unity. The short-dashed line shows the temporal pulse envelope. The inset shows the corresponding cut for the two-level atom model at the discrete line together with the corresponding pulse envelope (short dashes).

signal is completely dominated by the exciton response. The signal at the central laser frequency is more than four orders of magnitude smaller than at the exciton.

In Fig. 3 spectral cuts at the exciton are given. In this figure both data sets are normalized to unity for better comparison. In contrast to the narrow bandwidth case we find here a very different line shape for simulations with and without EID. The decay of the signal computed without EID [set (a)] is determined by the intrinsic dephasing rate $\gamma$, while the signal computed with EID [set (b)] has the temporal shape of the laser pulse, shown by the short-dashed line. The signal computed with EID increases slightly for delays $\left|\tau_{o}\right| \geqslant 48$ fs. At a delay of 48 fs the EID induced signal and the intrinsic signal are of comparable strength and they interfere destructively. At later times the EID contribution decays faster than the intrinsic one and the signal recovers be-

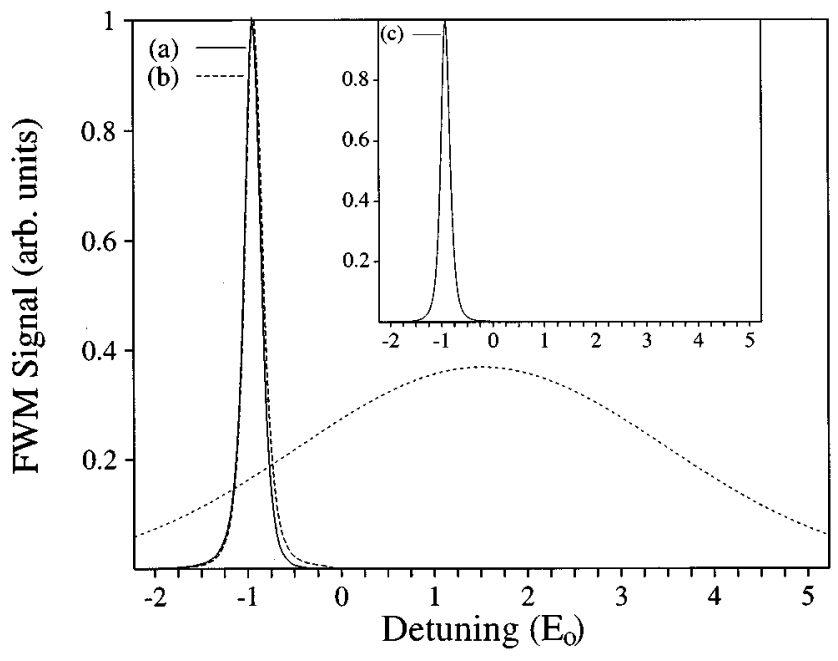

FIG. 4. Cuts from Fig. 2. FWM spectrum at zero delay versus detuning. The short-dashed line shows the spectral pulse profile. The inset shows the corresponding cut for the two-level atom model.

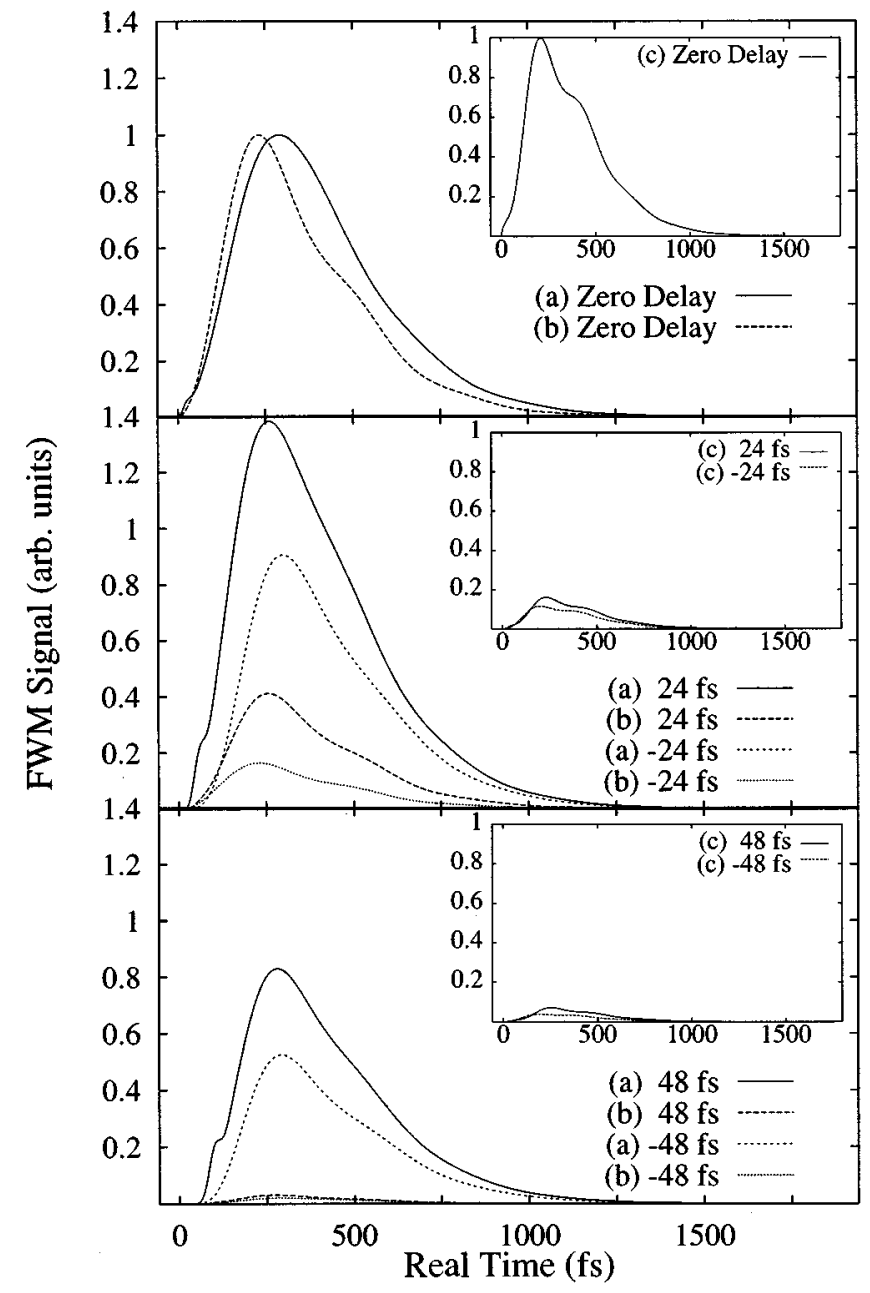

FIG. 5. Time-resolved FWM signal vs real time corresponding to Figs. 2(a) and 2(b). The inset gives the corresponding signal for the two-level atom model. The delay is zero (upper), $\pm 24 \mathrm{fs}$ (middle), and \pm 48 fs (lower).

fore it decays exponentially with the intrinsic dephasing time. This exponential decay occurs outside the shown delay time window.

The FWM spectra at zero delay are shown in Fig. 4. The extra dephasing caused by EID leaves the spectral shape of the excitonic response almost unchanged. The width is still determined by the intrinsic dephasing time. The short-dashed line gives the spectral profile of the laser pulse.

In Fig. 5, the corresponding temporally resolved FWM signals are shown for zero delay (upper), $\pm 24 \mathrm{fs}$ (middle), and for \pm 48 fs (lower). For better comparison, both data sets are scaled to yield a maximum of unity at zero delay. The relative strength of the signals computed with and without EID is again 35:1. The overall shape of the FWM signal versus real time is very similar for the parameter sets (a) and (b). They show the well-known features of signals from the nonlinearities due to the Coulomb exchange interaction. These features are (i) a maximum which is retarded in real time by about one dephasing time, (ii) an overall decay governed by the intrinsic dephasing rate, and (iii) signals of comparable strength for positive and negative delay (see, e.g., Refs. 2, 4, and 29.) The main difference between the 
signals computed with and without EID is the rapid decrease of the signal strength as a function of delay in the latter case.

We summarize the findings of this section in stating that (i) EID drastically enhances the FWM signal at zero delay, if together with the exciton a large number of continuum states is coherently excited, (ii) the coupling of the exciton to all the continuum states leads to a decay of the spectrally as well as the temporally resolved FWM signal, as a function of delay, on a time scale set by the pulse duration, (iii) the increased dephasing rate in a density maximum does not increase the width of the exciton line in the FWM spectrum, and (iv) the overall decay of the time-resolved FWM signal in real time is not affected. These findings agree well with the experimental data published in Ref. 10 for low-density excitations.

It is shown in the next section that all the mentioned features of the signals computed with EID can be understood in the framework of a simple two-level atom model.

\section{III. $\chi^{(3)}$ THEORY FOR AN ENSEMBLE OF TWO-LEVEL ATOMS WITH EXCITATION INDUCED DEPHASING}

In this section the influence of a density-dependent dephasing rate on the nonlinear optical properties is investigated in the frame of the following Bloch equation for an ensemble of two-level atoms:

$$
\begin{gathered}
\frac{d}{d t} P_{\lambda}(t)=-i\left[\epsilon_{\lambda}-i\left(\gamma_{\lambda}+\gamma^{\prime} \sum_{\mu} f_{1 \mu}(t)\right)\right] P_{\lambda}(t) \\
+i\left[1-2 f_{1 \lambda}(t)\right] d_{\lambda} E(t), \\
\frac{d}{d t} f_{1 \lambda}(t)=-2 \operatorname{Im}\left[d_{\lambda} E(t) P_{\lambda}^{*}(t)\right] .
\end{gathered}
$$

The two-level atoms are labeled by the index $\lambda$ and $\epsilon_{\lambda}=\epsilon_{1 \lambda}-\epsilon_{0 \lambda}$ is the energy difference between the two levels. The occupation of the lower level is $f_{0 \lambda}=1-f_{1 \lambda}$. Again, Eq. (3.1) takes excitation induced dephasing (EID) into account through the coefficient $\gamma^{\prime}$. ( $\hbar$ is taken to be unity in this section.)

Wang et al. presented in Ref. 12 a model which is similar to the one proposed in Eq. (3.1). The difference between the two models is the bandwidth of the EID induced coupling: Wang et al. use for EID the expression (translated in to the notation of this work) $\gamma^{\prime} f_{1 \lambda}$ instead of the EID coupling $\gamma^{\prime} \Sigma_{\mu} f_{1 \mu}$ proposed here, i.e., in the Wang model EID couples an excited state only to itself, while in our model EID couples each state to all other states. We feel that the model proposed here is more realistic, because EID is caused by scattering and an excited state is scattered by other states. This difference in the two models is crucial for the understanding of the mechanism which causes the CC. It is the coupling to a large number of states that causes $\mathrm{CC}$ and therefore the model presented by Wang et al., although similar, is not capable of describing CC.

From Eq. (3.1) analytic expressions for the nonlinear optical response on the $\chi^{(3)}$ level can be derived if the laser field $E(t)$ is taken to be the sum over two $\delta$ functions,

$$
E(t)=E_{1} e^{i \vec{K}_{1} \vec{R}} \delta(t)+E_{2} e^{i \vec{K}_{2} \vec{R}} \delta\left(t-\tau_{o}\right),
$$

where $\tau_{o}$ is the time delay and $\vec{K}_{1 / 2}$ denotes the propagation direction of the respective beams. In the following, the notation $\mathcal{E}_{1 / 2}$ is used for $E_{1 / 2} e^{i \vec{K}_{1 / 2} \vec{R}}$. The first-order polarization is given by

$$
P_{\lambda}^{(1)}(t)=\mathcal{P}_{\lambda}^{1}(t)+\mathcal{P}_{\lambda}^{2}(t)
$$

with

$$
\begin{gathered}
\mathcal{P}_{\lambda}^{1}(t)=i d_{\lambda} \mathcal{E}_{1} e^{-i\left(\epsilon_{\lambda}-i \gamma_{\lambda}\right) t} \Theta(t), \\
\mathcal{P}_{\lambda}^{2}(t)=i d_{\lambda} \mathcal{E}_{2} e^{-i\left(\epsilon_{\lambda}-i \gamma_{\lambda}\right)\left(t-\tau_{o}\right)} \Theta\left(t-\tau_{o}\right) .
\end{gathered}
$$

For the second-order density one finds

$$
f_{1 \lambda}^{(2)}(t)=\mathcal{F}_{\lambda}^{11}+\mathcal{F}_{\lambda}^{22}+\mathcal{F}_{\lambda}^{12}+\mathcal{F}_{\lambda}^{21}
$$

with

$$
\begin{gathered}
\mathcal{F}_{\lambda}^{11}=2 d_{\lambda}^{2}\left|\mathcal{E}_{1}\right|^{2} \Theta(t), \\
\mathcal{F}_{\lambda}^{22}=2 d_{\lambda}^{2}\left|\mathcal{E}_{2}\right|^{2} \Theta\left(t-\tau_{o}\right), \\
\mathcal{F}_{\lambda}^{12}=d_{\lambda}^{2} \mathcal{E}_{1} \mathcal{E}_{2}^{*} e^{-i \epsilon_{\lambda} \tau_{o}} e^{-\gamma_{\lambda}\left|\tau_{o}\right|} \\
\times\left[\Theta\left(\tau_{o}\right) \Theta\left(t-\tau_{o}\right)+\Theta\left(-\tau_{o}\right) \Theta(t)\right], \\
\mathcal{F}_{\lambda}^{21}=\left(\mathcal{F}_{\lambda}^{12}\right)^{*},
\end{gathered}
$$

where $\mathcal{F}_{\lambda}^{11}$ and $\mathcal{F}_{\lambda}^{22}$ describe the spatially homogeneous densities generated by the first and second pulses, respectively, and $\mathcal{F}_{\lambda}^{12}$ and $\mathcal{F}_{\lambda}^{21}$ describe the transient gratings set up by the interference pattern of the two beams. The first pulse sets up a polarization of the state $\lambda$, which oscillates and decays in time. After the time $\left|\tau_{o}\right|$ the second pulse converts the leftover polarization into an occupation. The transient grating therefore carries phase information. If the energy levels $\epsilon_{\lambda}$ are distributed, then the transient gratings associated with different states are in phase only for zero delay. For nonzero delay the phase is rapidly randomized. This additional inhomogeneous dephasing is the cause of fast decay of the CC.

The general third-order polarization is

$$
\begin{aligned}
P_{\lambda}^{(3)}(t)= & -\int_{-\infty}^{t} d t^{\prime} e^{-i\left(\epsilon_{\lambda}-i \gamma_{\lambda}\right)\left(t-t^{\prime}\right)}\left[2 i f_{1 \lambda}^{(2)}\left(t^{\prime}\right) d_{\lambda} E\left(t^{\prime}\right)\right. \\
& \left.+\gamma^{\prime} \sum_{\mu} f_{1 \mu}^{(2)}\left(t^{\prime}\right) P_{\lambda}^{(1)}\left(t^{\prime}\right)\right]
\end{aligned}
$$

Only the signal propagating in the $2 \vec{K}_{2}-\vec{K}_{1}$ direction contributes to the FWM signal, hence the third-order polarization reduces to

$$
\begin{aligned}
\left.P_{\lambda}^{(3)}(t)\right|_{\mathrm{FWM}}= & -\int_{-\infty}^{t} d t^{\prime} e^{-i\left(\epsilon_{\lambda}-i \gamma_{\lambda}\right)\left(t-t^{\prime}\right)} \\
& \times\left[2 i \mathcal{F}_{\lambda}^{21}\left(t^{\prime}\right) d_{\lambda} \mathcal{E}_{2} \delta\left(t^{\prime}-\tau_{o}\right)\right. \\
& \left.+\gamma^{\prime} \sum_{\mu} \mathcal{F}_{\mu}^{21}\left(t^{\prime}\right) \mathcal{P}_{\lambda}^{2}\left(t^{\prime}\right)\right]
\end{aligned}
$$




\section{A. Time-resolved FWM signals}

For the time-resolved FWM signal one finds

$$
\left.S_{\mathrm{FWM}}\left(\tau_{o}, t\right) \propto\left|\sum_{\lambda} d_{\lambda} P_{\lambda}^{(3)}\left(\tau_{o}, t\right)\right|_{\mathrm{FWM}}\right|^{2}
$$

with

$$
\begin{aligned}
d_{\lambda} P_{\lambda}^{(3)} & \left.\left(\tau_{o}, t\right)\right|_{\mathrm{FWM}} \\
= & -\mathcal{E}_{2}^{2} \mathcal{E}_{1}^{*} d_{\lambda}^{2}\left(\Theta\left(\tau_{o}\right) \Theta\left(t-\tau_{o}\right) 2 i d_{\lambda}^{2} e^{-\gamma_{\lambda}|t|}\right. \\
& \times e^{-i \epsilon_{\lambda}\left(t-2 \tau_{o}\right)}+\left[\Theta ( \tau _ { o } ) \left\{\Theta\left(t-\tau_{o}\right) e^{-\gamma_{\lambda}\left|t-\tau_{o}\right|}\right.\right. \\
& \left.\left.\times\left(t-\tau_{o}\right)\right\}+\Theta\left(-\tau_{o}\right) e^{-\gamma_{\lambda}\left|\tau_{o}\right|}\left\{\Theta(t) e^{-\gamma_{\lambda}|t|} t\right\}\right] \\
& \left.\times i \gamma^{\prime} e^{-i \epsilon_{\lambda}\left(t-\tau_{o}\right)} F\left(\tau_{o}\right)\right)
\end{aligned}
$$

and

$$
F\left(\tau_{o}\right)=\sum_{\mu} d_{\mu}^{2} e^{i \epsilon_{\mu} \tau_{o}} e^{-\gamma_{\mu}\left|\tau_{o}\right|}
$$

The phase space filling (PSF) contribution, i.e., the first term in Eq. (3.9), gives the well-known free polarization decay for a discrete state and the so-called "photon echo" for an inhomogenously broaden continuum of two-level atoms. ${ }^{31}$ The PSF contribution stems from the transient gratings induced by the interference pattern of the two laser beams: The grating scatters a part of the second pulse in the FWM direction.

The process behind the EID contribution [second term in Eq. (3.9)] is similar to the one discussed above, except that (i) in this case the polarization set up by the second pulse is scattered in the FWM direction. Because the polarization exists in the sample on a time scale set by the dephasing rate, the signal contributes for positive and negative delay and (ii) EID provides a broad-band coupling to all states. Therefore, the EID signal in Eq. (3.7) contains the coherent superposition $\Sigma_{\mu} \mathcal{F}_{\mu}^{21}$ of the transient gratings associated with all excited states described by $F\left(\tau_{o}\right)$ in Eq. (3.9).

For a more detailed discussion of the signals we assume that the energy separations $\epsilon_{\lambda}$ of the two-level atoms are distributed as follows. The state $\lambda=0$ is discrete, while all other states $\lambda>0$ merge into a continuum that extends from $\epsilon_{1}=\epsilon_{g}$ to infinity and are described by a density of states $\rho(\epsilon)$. In order to model the finite spectral width of an actual laser pulse we take the density of states to be

$$
\rho(\epsilon)=\frac{\Theta\left(\epsilon-\epsilon_{1}\right)}{\Delta \epsilon} e^{-\left(\epsilon-\epsilon_{1}\right) / \sigma} .
$$

The parameter $\Delta \epsilon$ can be regarded as the effective level spacing, while $\sigma$ simulates the effect of a finite spectral width of a (Lorentzian) laser pulse. For a constant oscillator strength $d_{c}$ and dephasing rate $\gamma_{c}$ in the continuum, $F\left(\tau_{o}\right)$ becomes

$$
\begin{aligned}
F\left(\tau_{o}\right) & =d_{0}^{2} e^{-\gamma_{0}\left|\tau_{o}\right|} e^{i \epsilon_{0} \tau_{o}}+d_{c}^{2} e^{-\gamma_{c}\left|\tau_{o}\right|} \int_{\epsilon_{g}}^{\infty} d \epsilon g(\epsilon) e^{i \epsilon \tau_{o}} \\
& =d_{0}^{2} e^{-\gamma_{0}\left|\tau_{o}\right|} e^{i \epsilon_{0} \tau_{o}}+d_{c}^{2} e^{-\gamma_{c}\left|\tau_{o}\right|} e^{i \epsilon_{g} \tau_{o}} \frac{\sigma}{\Delta \epsilon} \frac{1}{1+i \sigma \tau_{o}}
\end{aligned}
$$

The ratio $N_{\text {eff }}=\sigma / \Delta \epsilon$ is the effective number of excited continuum states. For an excitation with a spectrally broad laser pulse $N_{\text {eff }}$ can be large enough to overcompensate that the continuum oscillator strength $d_{c}$ is smaller than the one of the discrete state $d_{0}$. In turn the EID induced contribution can be much stronger than the PSF signal. Due to the coherent superposition of the transient gratings associated with the different continuum states $F\left(\tau_{o}\right)$ decays quickly. The decay becomes faster when more continuum states are excited (see Figs. 2 and 3). This analysis shows that EID enhances the time-resolved FWM signal for small delays, but for a fixed delay the FWM signal still decays with the intrinsic decay rate $\gamma_{\lambda}$ in real time. This explains the behavior seen in Fig. 5.

A further characteristic feature of the EID term is the function $\Theta(T) T e^{-\gamma|T|}$, with $T$ being either $t$ or $t-\tau_{o}$, which reaches its maximum at $T=1 / \gamma$. This "interaction retardation', is discussed, e.g., in Refs. 2-4 and 29. Its Fourier transform produces a squared Lorentzian in the spectrally resolved signals [see below in Eq. (3.14)].

\section{B. Spectrally resolved FWM signals}

The corresponding spectrally resolved signal is given by

$$
\left.S_{\mathrm{FWM}}\left(\tau_{o}, \omega\right) \propto\left|\sum_{\lambda} d_{\lambda} P_{\lambda}^{(3)}\left(\tau_{o}, \omega\right)\right|_{\mathrm{FWM}}\right|^{2}
$$

with

$$
\begin{aligned}
\left.d_{\lambda} P_{\lambda}^{(3)}\left(\tau_{o}, \omega\right)\right|_{\mathrm{FWM}} \\
=\mathcal{E}_{2}^{2} \mathcal{E}_{1} d_{\lambda}^{2}\left(-\Theta\left(\tau_{o}\right) 2 d_{\lambda}^{2} e^{-\gamma_{\lambda}\left|\tau_{o}\right|} \frac{e^{i\left(\epsilon_{\lambda}+\omega\right) \tau_{o}}}{\epsilon_{\lambda}-\omega-i \gamma_{\lambda}}\right. \\
\quad+\left[\Theta\left(\tau_{o}\right) e^{i \omega \tau_{o}}+\Theta\left(-\tau_{o}\right) e^{-\gamma_{\lambda}\left|\tau_{o}\right|} e^{i \epsilon_{\lambda} \tau_{o}}\right] i \gamma^{\prime} \\
\left.\quad \times \frac{F\left(\tau_{o}\right)}{\left(\epsilon_{\lambda}-\omega-i \gamma_{\lambda}\right)^{2}}\right)
\end{aligned}
$$

The PSF contribution, i.e., the first term in Eq. (3.14), closely resembles the linear absorption spectrum at $\tau_{o}=0$. For nonzero delay the additional phase factors produce beats between characteristic energies in the ensemble, e.g., the discrete state and the band edge. As discussed above, the EID terms are proportional to a squared Lorentzian. This function is responsible for the spectral narrowing reported in Ref. 7 and explains why the additional dephasing induced by EID does not widen the excitonic line in Fig. 4.

The squared Lorentzian has another important implication. For energies within the continuum the EID induced signal contains the expression

$$
\int_{\epsilon_{g}}^{\infty} d \epsilon \frac{\rho(\epsilon)}{\left(\epsilon-\omega-i \gamma_{c}\right)^{2}} \sim \rho(\omega) \int_{-\infty}^{\infty} d \epsilon \frac{1}{\left(\epsilon-\omega-i \gamma_{c}\right)^{2}}=0
$$


For a frequency $\omega$ which is several $\gamma_{c}$ above the band edge $\epsilon_{g}$, the integration range can be extended to $-\infty$. For an excitation with an ultrashort laser pulse, $\rho(\epsilon)$ varies only little in the $\epsilon$ range which contributes to the integral and therefore can be replaced by $\rho(\omega)$. The remaining integral over the squared energy denominator vanishes (a pole of order 2 has no residue). The physical interpretation is that the overlapping continuum states give contributions which interfere destructively and the EID signal is important only at a discrete state and at the band edge. This explains the absence of a signal at the spectral position of the resonantly excited continuum states in Figs. 2 and 4.

\section{C. $\chi^{(3)}$ results and discussion}

Figure 2(c) and the insets in Figs. 3-5 illustrate the signals computed from Eqs. (3.8) and (3.13) for parameters comparable with set (b) from Sec. II, i.e., $\epsilon_{0}=-E_{o}$, $\epsilon_{g}=0, \quad \gamma_{0}=\gamma_{c}=1 / 250 \quad$ fs, $\quad \gamma^{\prime}=3 \times 10^{-13} \mathrm{~cm}^{2} / \mathrm{fs}$, $\sigma=3.75 E_{o}$, and $\Delta \epsilon=0.05 E_{o}$. The ratio of the oscillator strength of the discrete state to that of the continuum states $d_{0} / d_{c}=50$ was chosen such that the linear absorption spectra resembles the one for sets (a) and (b).

The two-level atom model with EID shows the same features as the numerical solutions of the SBE with EID, although the structure of the two models is quite different. Formally, the SBE [Eq. (2.1)] reduce to Eq. (3.1) if one neglects all terms containing the Coulomb potential $V_{q}$ but retains, nevertheless, the EID. It is well-known, however, that a free-particle picture is not adequate to model the optical response of a semiconductor near the band gap. ${ }^{22}$ The correlations of the excited electron-hole pairs induced by the local field transform the absorption spectrum from a freecarrier step function (in 2D) into an excitonic series. In order to derive simple analytic results we chose to retain the simplicity of a two-level atom model, but nevertheless capture the main aspects of the full SBE which lead to CC. We therefore mimicked the shape of the excitonic absorption spectrum by assuming a "custom-tailored" distribution of two-level atoms [see Eq. (3.11) and text above]. A more rigorous way would be to formulate the optical response in an excitonic basis. This, however, is a delicate task, because excitons are not real bosons and much work has been devoted to that problem. ${ }^{32}$ The model proposed in Eq. (3.1) has far to many free parameters to be used as a predictive tool, but in combination with the full SBE it provides a simple analytical understanding to the influence of EID on the FWM line shape. We showed that in the limit of a broad band excitation EID completely dominates the FWM signal. The proposed model, Eqs. (3.1), focuses on this aspect and neglects others, e.g., the Coulomb exchange interaction or the partially bosonic nature of the fundamental excitations which are of minor importance in this particular respect. Furthermore, we would like to point out that the analytic expressions given above, describe some general aspects of interaction induced FWM signals. For example, if one lets $\gamma^{\prime}$ be imaginary the EID term in Eq. (3.1) describes the band gap renormalization induced by a point interaction. Also the retardations discussed above are common to all types of interaction induced signals.

\section{DISCUSSION AND CONCLUSIONS}

It was shown that excitonic FWM signals are dominated by a component which decays as a function of delay on the time scale of the exciting laser pulse, if a large number of continuum states is excited coherently together with the exciton. At the same time virtually no signal was observed at the spectral position of the continuum states themselves. This effect was explained by assuming a broad-band coupling of the exciton to the continuum states. We demonstrated how this coupling leads, in a nonlinear optical experiment, to signals from all states at the spectral position of all other states. These signals add coherently and in an inhomogeneous system the total signal decays very fast as a function of delay time. We showed that in the continuum, there is an additional destructive interference of signals from neighboring states. Due to this destructive interference, the signal from this nonlinearity can only be seen at a discontinuity of the density of states, like the exciton or the band edge. We discussed how a broad-band coupling arises from scattering. We showed a simple method of modeling such a coupling in the SBE by extending the $T_{2}$ model of the polarization decay to include a density-dependent dephasing rate. It was shown that the effect of the broad-band coupling can be understood in a two-level atom model with one discrete state interacting with an ensemble of other two-level atoms.

We end this section with a discussion of recent related work. Kim et al. ${ }^{11}$ reported a so-called "giant" excitonic resonance in FWM experiments of GaAs quantum wells in the low-density regime. The authors simulated the experiment using the SBE assuming a density independent dephasing rate. In order to explain their experimental data, they assumed very different dephasing rates for the exciton $(800$ fs) and the continuum (80 fs) and concluded that in the FWM signal the state with the longest dephasing time dominates, and that even in the continuum the signal decays with the excitonic decay time. In the present paper we have shown that the enhancement of the excitonic signal can be explained through EID, and that within this framework the assumption of a very rapid dephasing of the continuum states is not necessary. It was shown that the decay of the signal in the continuum is related to the intrinsic dephasing time of the continuum states.

Rappen et al. ${ }^{13}$ reported FWM simulations based on the SBE with Boltzmann-like scattering integrals in the equations of motion of the polarization and the occupation numbers. They found that one of the effects of the scattering integrals in the polarization equation is to transfer oscillator strength from the continuum to the exciton. This is in agreement with the finding of this paper in which the effects of the polarization scattering integral was modeled phenomenologically by the EID concept.

\section{ACKNOWLEDGMENTS}

K.E.S. acknowledges support from the National Science Foundation through Grant No. DMR-9520191. V.G.L. acknowledges support from the Danish Ministries of Industry and Research in the framework of CNAST. 


\section{APPENDIX: MOTIVATION OF THE EID APPROACH}

Here we discuss the microscopic theory of the scattering integrals in the polarization equation. For times during and shortly after the laser pulses excite the sample one has to use quantum kinetic expressions. Quantum kinetic scattering integrals on the RPA level can be derived using Keldysh nonequilibrium Green functions (see, e.g., Refs. 24 and 33). In the low-density limit $\left(f_{j k} \ll 1\right)$ one finds ${ }^{34}$

$$
\begin{aligned}
\left.\frac{\partial}{\partial t} P_{k}(t)\right|_{\text {scatt }}= & -\frac{2}{\hbar} \int_{\infty}^{t} d t^{\prime} \sum_{\mathbf{p q}} V_{q}^{2}\left(P_{k}-P_{|\mathbf{k}-\mathbf{q}|}\right) \\
& \times \operatorname{Re}\left[\sum_{j=e, h}\left(f_{j p}-P_{|\mathbf{p}+\mathbf{q}|} P_{p}^{*}\right)\right. \\
& \left.\times e^{-i / \hbar\left(\epsilon_{j|\mathbf{p}+\mathbf{q}|}-\epsilon_{j p}+\epsilon_{e|\mathbf{k}-\mathbf{q}|}+\epsilon_{h k}\right)\left(t-t^{\prime}\right)}\right] .
\end{aligned}
$$

It was shown in Ref. 35 that it takes a time of about one characteristic inverse plasma frequency after the excitation by the ultrashort laser pulse before screening sets in. The Coulomb potential in Eq. (A1) is therefore unscreened. In the low-density regime, screening is not efficient even at later times.

At later times, when the system is already near equilibrium, the occupation numbers and the polarization will vary only slightly over the range of the memory integration in Eq. (A1). The time integration over the remaining phase factors gives an energy denominator. Its imaginary part is a (broadened) energy conserving $\delta$ function known from Boltzmann scattering integrals. The real part describes additional energy renormalizations due to scattering. At this stage screening has been established and the bare Coulomb potential is to be replaced by a (weakly) screened one.

In both the quantum kinetic and the Boltzmann regime, " out" $\left(\propto P_{k}\right)$ and "in" scattering rates $\left(\propto P_{|\mathbf{k}-\mathbf{q}|}\right)$ cannot be defined separately. In the quantum kinetic regime the divergence of the bare Coulomb potential in the limit $q \rightarrow 0$ is compensated only by the term $\left(P_{k}-P_{|\mathbf{k}-\mathbf{q}|}\right)$ and even in the Boltzmann regime for low-density systems it is known that a considerable compensation between "out" and "in" scattering occurs. This shows that a direct derivation of an expression for a density-dependent ("out") scattering rate like the one assumed in Eq. (2.6) is not possible. Nevertheless, the expression (A1) describes an effective dephasing which is linear in the density. ${ }^{24} \mathrm{~A}$ state $\mathbf{k}$ is coupled to all other states p through a density-independent coupling constant. Only through screening the coupling becomes density-dependent. The approximation (1.1) simply simulates these two aspects: (i) a broad-band coupling to all other states and (ii) a linear density dependence.
*Permanent address: Institute of Microelectronics Technology and Superpure Materials, Chernogalova, Moscow District, 142432, Russia.

${ }^{1}$ H. Haug and S. Schmitt-Rink, Prog. Quantum Electron. 9, 3 (1984).

${ }^{2}$ M. Lindberg, R. Binder, and S.W. Koch, Phys. Rev. A 45, 1865 (1992).

${ }^{3}$ T. Rappen, U. Peter, M. Wegener, and W. Schäfer, Phys. Rev. B 48, 4879 (1993).

${ }^{4}$ D.-S. Kim, J. Shah, T. Damen, W. Schäfer, F. Jahnke, S. SchmittRink, and K. Köhler, Phys. Rev. Lett. 69, 2725 (1992).

${ }^{5}$ W. Schäfer, F. Jahnke, and S. Schmitt-Rink, Phys. Rev. B 47, 1217 (1993).

${ }^{6} \mathrm{~A}$ (close to) band-width limited ultrashort laser pulse has sufficient coherent band width to excite excitons and continuum states coherently, if tuned in the vicinity of the band edge.

${ }^{7}$ D. Birkedal, V.G. Lyssenko, J.M. Hvam, and K. El Sayed, Phys. Rev. B 54, 14250 (1996).

${ }^{8}$ J. Feldmann, T. Meier, G. von Plessen, M. Koch, E. Göbel, P. Thomas, G. Bacher, C. Hartmann, H. Schweizer, W. Schäfer, and H. Nickel, Phys. Rev. Lett. 70, 3027 (1993).

${ }^{9}$ J. Erland, D. Birkedal, V. G. Lyssenko, and J. M. Hvam, J. Opt. Soc. Am. B 13, 981 (1996).

${ }^{10}$ M.U. Wehner, D. Steinbacher, M. Wegener, T. Marschner, and W. Stolz, J. Opt. Soc. Am. B 13, 977 (1996).

${ }^{11}$ D.-S. Kim, J. Shah, J.E. Cunningham, T.C. Damen, W. Schäfer, M. Hartmann, and S. Schmitt-Rink, Phys. Rev. Lett. 68, 1006 (1992).

${ }^{12}$ H. Wang, K.B. Ferrio, D.G. Steel, P.R. Berman, Y. Hu, R. Binder, and S.W. Koch, Phys. Rev. A 49, R1551 (1994).

${ }^{13}$ T. Rappen, U.-G. Peter, M. Wegener, and W. Schäfer, Phys. Rev. B 49, 10774 (1994).
${ }^{14}$ L. Schultheis, J. Kuhl, A. Honold, and C.W. Tu, Phys. Rev. Lett. 57, 1635 (1986).

${ }^{15}$ A. Honold, L. Schultheis, J. Kuhl, and C.W. Tu, Phys. Rev. B 40, 6442 (1989).

${ }^{16}$ In the later experiment the laser creates a (partially) coherent system, i.e., the induced microscopical polarization and the carrier occupation numbers have a phase relation, while in the prepump experiments the two quantities are independent.

${ }^{17}$ H. Wang, K. Ferrio, D.G. Steel, Y.Z. Hu, R. Binder, and S.W. Koch, Phys. Rev. Lett. 71, 1261 (1993).

${ }^{18}$ Y. Hu, R. Binder, S.W. Koch, S. Cundiff, H. Wang, and D.G. Steel, Phys. Rev. B 49, 14382 (1994).

${ }^{19}$ S. Schmitt-Rink, S. Mukamel, K. Leo, J. Shah, and D.S. Chemla, Phys. Rev. A 44, 2124 (1991).

${ }^{20}$ S. Schmitt-Rink and D.S. Chemla, Phys. Rev. Lett. 57, 2752 (1986); S. Schmitt-Rink, D.S. Chemla, and H. Haug, Phys. Rev. B 37, 941 (1988).

${ }^{21}$ M. Lindberg and S.W. Koch, Phys. Rev. B 38, 3342 (1988).

${ }^{22}$ H. Haug and S. W. Koch, Quantum Theory of the Optical and Electronic Properties of Semiconductors, 2nd ed. (World Scientific, Singapore, 1993).

${ }^{23}$ D.B. Tran Thoai and H. Haug, Z. Phys. B 91, 199 (1993); 92, 532 (1993).

${ }^{24}$ K. El Sayed, L. Bányai, and H. Haug, Phys. Rev. B 50, 1541 (1994).

${ }^{25}$ D. Tran Thoai, L. Bányai, E. Reitsammer, and H. Haug, Phys. Status Solidi B 188, 387 (1995).

${ }^{26}$ D.C. Scott, R. Binder, and S.W. Koch, Phys. Rev. Lett. 69, 347 (1992).

${ }^{27}$ R. Binder, D.C. Scott, A. Paul, M. Lindberg, K. Henneberger, and S.W. Koch, Phys. Rev. B 45, 1107 (1992).

${ }^{28}$ L. Bányai, D.B. Tran Thoai, E. Reitsammer, H. Haug, D. Stein- 
bacher, M.U. Wehner, M. Wegener, T. Marschner, and W. Stolz, Phys. Rev. Lett. 75, 2188 (1995).

${ }^{29}$ M. Wegener, D. Chemla, S. Schmitt-Rink, and W. Schäfer, Phys. Rev. A 42, 5675 (1990).

${ }^{30}$ For this parameter set the additional dephasing induced by EID is small compared to the intrinsic one, $\gamma^{\prime} n \ll \gamma$, and an adjustment of $\gamma$ for the simulations with and without EID is unnecessary.

${ }^{31}$ P. Meystre and M. Sargent III, Elements of Quantum Optics (Springer, Berlin, 1991).
${ }^{32}$ See, e.g., H. Stolz, R. Zimmermann, and G. Röpke, Phys. Status Solidi B 105, 585 (1981).

${ }^{33} \mathrm{H}$. Haug and A. Jauho, Quantum Kinetics in Transport and Optics of Semiconductors (Springer, Heidelberg, 1996).

${ }^{34}$ In order to get a concise expression it is assumed that the polarization and the occupation numbers depend only on the absolute value of the wave vector.

${ }^{35}$ K. El Sayed, S. Schuster, H. Haug, F. Herzel, and K. Henneberger, Phys. Rev. B 49, 7337 (1994). 